\title{
Gait Generation via the Foot Placement Estimator for 3D Bipedal Robots
}

\author{
Safwan Choudhury \\ Electrical and Computer Engineering \\ University of Waterloo. Waterloo, ON, Canada \\ schoudhu@uwaterloo.ca
}

\author{
Dana Kulić \\ Electrical and Computer Engineering \\ University of Waterloo. Waterloo, ON, Canada \\ dkulic@uwaterloo.ca
}

\begin{abstract}
This paper proposes a trajectory generation and control strategy for generating stable gait subject to unknown disturbances, based on the concept of the Foot Placement Estimator (FPE). While most walking control strategies in the field of bipedal locomotion aim to constantly maintain balance, the Foot Placement Estimator (FPE) estimates where the foot must be placed in order to restore balance. One of the key novelties of the FPE approach is its natural extension to form complete gait cycles using a state machine and simple proportional-derivative controllers. In this paper, the FPE control strategy is extended from $2 D$ to $3 D$ robots, and demonstrated in simulation on a 14-DOF lower body bipedal robot.
\end{abstract}

Index Terms-Bipedal Locomotion, Foot Placement, Gait Synthesis.

\section{INTRODUCTION}

Maintaining balance is one of the key challenges inherent to the bipedal form. Most traditional approaches have been based on the concept of constantly maintaining balance [1], [2]. The most popular techniques to achieve walking have been trajectory generation and control strategies based on the Zero-Moment Point (ZMP) criterion [3]. The ZMP defines a point on the ground where the forces acting on a biped do not produce a moment about the axes parallel to the ground plane. If the ZMP is kept within the region of foot support, the biped is stable. This stability criterion can be used to compute ZMPstable trajectories offline that maximize the stability margin by maximizing the distance from the ZMP to the boundary of the support region. In the on-line phase, the stable trajectories are tracked online to execute walking [4]. ZMP-based trajectories can also be generated on-line [5], [6]. One of the biggest drawbacks of this approach is the resulting trajectory does not provide any strategy to respond to disturbances due to uneven terrain or unexpected forces. Typically, these strategies [7], [2] are also energetically inefficient since they are constantly trying to maintain balance by keeping the ZMP point within the region of foot support.

An alternative approach, first proposed by McGeer [8], introduced a unique class of legged robots known as passive dynamic walkers [9]. This approach takes advantage of the natural dynamics of the biped structure, and is capable of maintaining a stable gait cycle without active control effort. Fully passive mechanisms walk on an inclined surface so that the mechanism is powered by gravity alone [10]. In addition to producing highly efficient walk, the gait patterns generated using this approach appear more human-like in comparison to ZMP-based control. However, passive dynamic walkers lack robustness to perturbations due to very narrow regions of attraction.

Recently, an alternative problem formulation focusing on restoring balance has been proposed. The Foot Placement Estimator (FPE), introduced by Wight et al. [11] formulates an approach to restore balance by controlling swing foot position during the gait cycle. By using the conservation of angular momentum, the FPE equation determines the location on the ground where the total energy of an unstable biped after swing foot impact is equal to the peak potential energy. If a step is taken before the FPE location, the post impact energy of the system causes the biped to fall over. Conversely, stepping beyond the FPE location on the ground causes the biped to fall back onto the previous stance leg.

The solution to the FPE equation itself can be used as a recovery mechanism (i.e. in the face of a destabilizing disturbance) with existing ZMP-based strategies. Alternatively, it can be used to increase the narrow regions of attraction which plague minimally actuated passive dynamic walkers [12], [13], [14]. The key concept here is that the FPE-based integration would require minimal joint actuation only to align a swing food appropriately to recover from a potential fall.

As shown in [11], [15], FPE can also be extended to form complete gait cycles to achieve dynamically stable walking. However, there are several key assumptions which are violated when attempting to implement this approach on a physical 3D robot. Namely, the theory presented in the derivations assumes that the legs are massless and it only deals with the 2D dynamics in the sagittal plane.

In this paper, we extend the 2D FPE theory for 3D bipedal robots by selecting a plane of motion in 3D space along the desired direction of movement and constraining the swing foot motion within this plane. Once the biped is unstable, we solve FPE equation along the selected plane to determine the swing foot placement to ultimately regain stability. Similar to the approach used in [11], [15], we extend this concept to form complete gait cycles with a state machine, high gain PD controllers and no precomputed trajectories.

The capture point concept, developed by Pratt et al. [16], is conceptually similar to the FPE. While the derivation of FPE is based on a simple compass biped model with 


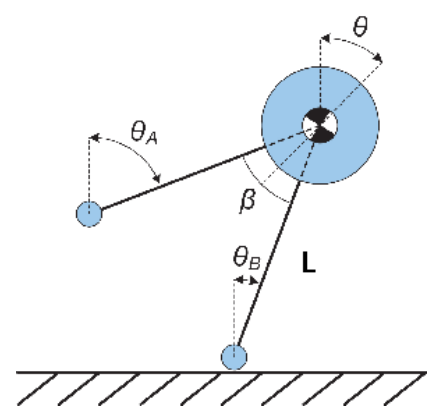

Fig. 1. Unified variable $\theta$ used to simplify the analysis. It is easily observed that $\theta_{A}=\theta+\beta / 2$ and $\theta_{B}=\theta-\beta / 2$

fixed parameters, the capture point theory was derived using complex motion models which included using a flywheel body to control/offset any disturbances through the use of rotational inertia. Ultimately, the simplicity of the model allowed the FPE theory to be extended to complete gait cycles, while the work presented by Pratt et al simply solved the problem of lateral stabilization.

Recently, a more comprehensive approach using the capture point for foot placement as a means to develop full walking control strategies has been proposed. De Boer [17] focused on the ground/foot interaction to develop a robust and energetically efficient walking control strategy for a force-controlled compliant lower-body biped. While this approach is philosophically similar to the idea behind FPE, there are several key differences. Our approach uses simple local controllers to form complete gait cycles, and can be used on positioncontrolled joints without any complex actuation systems. The capturability framework demonstrated in [18] used separate controllers for the swing and stance legs whereas our approach uses a single global differential kinematic resolution for whole body motion control. This simplifies our approach as multiple controllers do not need to be tuned to achieve walking.

The rest of this paper is organized as follows. Section II briefly reviews the underlying FPE theory developed for the $2 \mathrm{D}$ case. Section III proposes an approach for extending the FPE approach to 3D along with a proposed control strategy which closely resembles the $2 \mathrm{D}$ case. Section IV details simulations and results from various experiments with the 2D/3D FPE. Section V provides concluding remarks and directions for future work.

\section{Foot Placement Estimator in 2D}

In this section, the FPE approach for 2D systems initially developed by Wight et al. [11] is briefly reviewed. The derivation of the underlying theory begins with the simple biped model as shown in Figure 1. The physical parameters are the mass $m$, inertia about the center of mass $I_{C O M}$, fixed leg lengths $L$ and leg separation angle $\beta$. By imposing the geometric constraints that $\beta$ is fixed and there is no slipping at the contact points, a single state variable $\theta$ is used to completely define the motion of the compass biped:

$$
\ddot{\theta}=\left\{\begin{array}{cl}
\frac{m g L \sin (\theta+\beta / 2)}{I_{C O M}+m L^{2}} & \theta<0 \\
\frac{m g L \sin (\theta-\beta / 2)}{I_{C O M}+m L^{2}} & \theta>0 \\
\frac{m g L \sin (\theta+\beta / 2)}{I_{C O M}+m L^{2}} & \theta=0, \dot{\theta}>0 \\
\frac{m g L \sin (\theta-\beta / 2)}{I_{C O M}+m L^{2}} & \theta=0, \dot{\theta}<0 \\
0 & \theta=0, \dot{\theta}=0
\end{array}\right.
$$

The impact of the contact points with the ground is modelled using the conservation of angular momentum to determine the regions in the state space where the biped remains stable after ground contact, by analyzing the total system energy post impact. The stability analysis is then used to determine where a biped must step to remain within the stability region. This forms the basis of the FPE, which is a point on the ground where, if the robot were to step onto that point, the kinetic energy of the biped system would equal the peak potential energy. Placing the swing foot beyond the FPE point results in the biped not having enough kinetic energy post impact to overcome the peak potential energy. In this case, the biped remains stable. Conversely, placing the swing foot before the FPE point causes the kinetic energy post impact to exceed the peak potential energy. In this situation the biped begins to fall over.

\section{A. Computing the FPE Angle}

Here, $v_{x}$ and $v_{y}$ are the $x$ and $y$ components of velocity of the centre of mass, respectively. The FPE point is obtained by solving the non-linear FPE equation in (2) for the angle $\phi$. A projection from the COM to the ground surface at the angle $(\phi)$ provides the location of where the foot needs to be placed in order to restore stability to the unbalanced system (as shown in Figure 2).

While the stability analysis presented in [11] considered a simple compass biped, the results of the FPE equation can be extended to any arbitrary planar biped (i.e. with bent knees) by controlling the joint angles of the leg to achieve an equivalent distance $L$ between the COM and the foot contact. If a biped is unstable and begins to pivot forward on a fixed contact point, tracking the angle $\phi$ with the swing foot as the robot falls forward converges to the FPE location when the swing foot hits the ground. Upon impact, the distance between the contact point and the COM would have the the same fixed length $L$ between the contact points and the angle $\phi$ converges to $\beta / 2$. The results from the compass biped also extend to the arbitrary biped configuration (i.e. stepping directly on the FPE point results in the kinetic energy being equivalent to the peak potential energy).

\section{B. Forming Complete Gait Cycles}

Wight et al. [11] use the FPE concept to develop full gait cycles using simple linear control techniques and a state machine. Gait is initiated by destabilizing the robot in the 


$$
\frac{\left[m h\left(v_{x} \cos \phi+v_{y} \sin \phi\right) \cos \phi+I_{C O M} \dot{\theta}_{1} \cos ^{2} \phi\right]^{2}}{m h^{2}+I_{C O M} \cos ^{2} \phi}+2 m g h \cos \phi(\cos \phi-1)=0
$$

desired direction of movement (forward or backward). Once destabilized, the FPE equation is solved numerically to obtain the FPE angle $\phi$, which is used to provide the desired trajectory for the swing foot. If continued forward progress is desired, the foot is commanded to precede the FPE. If no further forward progress is needed, the foot is commanded to the FPE. The desired trajectory is resolved to joint angles using inverse kinematics and implemented via joint level PD controllers. The complete state machine is shown in Figure 3.

Due to symmetry, the states in Figure 3 can be reduced to STAND, PUSH, LIFT, SWING and DROP. For the remainder of this paper, we refer to the sequence of state transitions from PUSH to DROP as a step cycle.

\section{EXTENSION TO 3D}

In order to extend the FPE approach to the 3D case, we revisit the concept of generating complete gait cycles described in Section II-B. The primary goal of the first three states in each step cycle (PUSH, LIFT and SWING) is to force the biped into an unstable configuration so that the FPE algorithm can be used to regain stability in the terminal state (DROP).

To extend the $2 \mathrm{D}$ algorithm to the general $3 \mathrm{D}$ case, we begin by selecting a suitable plane in 3D space as the plane of motion. The non-motion plane is perpendicular to the plane of motion and the ground. In the proposed approach, the goal of each step cycle is to control the motion of a 3D bipedal robot to generate a forward moving momentum along the selected plane of motion. Upon entering the terminal state, we solve (2) on the selected plane to determine the swing foot placement and ultimately regain stability. Unlike the 2D case, we consider a 3D bipedal robot with finite foot length and width rather than a biped with point feet as demonstrated in [11]. The larger size of the region of support increases robustness to these approximation errors.

The remainder of this paper assumes a 3D bipedal robot with $n$ actuated degrees of freedom (DOF) and $n+6$ generalized coordinates defined by the following equation of motion:

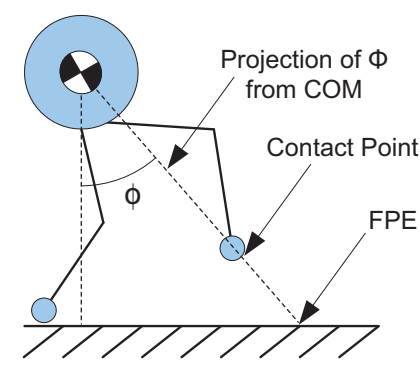

Fig. 2. Graphical representation of the solution to the FPE equation for arbitrary robot configurations.

$$
A(\mathbf{q}) \ddot{\mathbf{q}}+C(\mathbf{q}, \dot{\mathbf{q}}) \dot{\mathbf{q}}+g(\mathbf{q})=\tau
$$

where $A(\mathbf{q})$ is the $(n+6) \times(n+6)$ inertia matrix, $C(\mathbf{q}, \dot{\mathbf{q}})$ are the centripetal/Coriolis terms and $g(\mathbf{q})$ is the gravity vector. The $(n+6) \times 1$ generalized force vector $\tau$ is segmented as follows:

$$
\tau=\left[\begin{array}{ll}
\tau_{\text {act }} & \tau_{\text {base }}
\end{array}\right]^{T}
$$

Where $\tau_{a c t}$ represents the $n$ actuated DOF and $\tau_{\text {base }}=$ $\mathbf{0}_{\mathbf{6} \times \mathbf{1}}$ are the remaining non-actuated DOF at the floating base.

\section{A. Plane of Motion}

To select an appropriate plane of motion for a 3D bipedal robot, we choose a vertical plane which lies between the current position of the stance foot and the desired direction of motion. For a 3D biped walking in a forward motion, this plane is chosen as the the vertical plane passing through the midpoint between the hips and parallel to the direction of forward progress. For side-stepping motion, the coronal plane through the stance foot in the direction of the side step is chosen as the plane of motion.

The motion of the biped is controlled based on the selected plane for the duration of the step cycle. During gait initiation, the lines from the COM to the contact points are of length $L$, and the leg separation angle is $\beta$ (similar to the planar case). If the motion of the biped is constrained along this plane, the FPE angle $\phi$ can be used to determine foot placement to regain stability. The parameters required to compute the $2 \mathrm{D}$ FPE along the plane are computed accordingly. That is, the inertia tensor of each link is rotated to obtain the appropriate $I_{C O M}$ along the selected plane of motion.

Upon impact, the angle $\phi$ converges to $\beta / 2$ and a new plane of motion can be selected for the subsequent step cycle, at

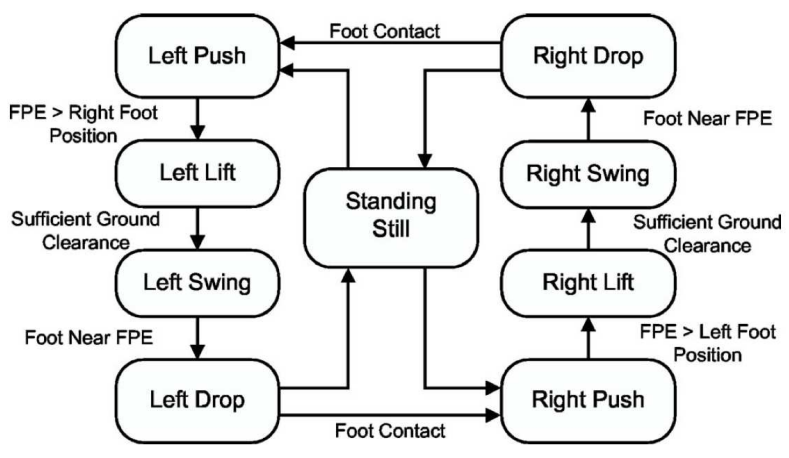

Fig. 3. A simple state machine used in conjunction with the FPE algorithm to form complete gait cycles 
any point before the DROP state. Once selected, the stance foot is rotated for alignment and swing leg trajectories can be generated along the plane. By selecting a plane between the current and desired directions of motion, our approach can achieve turning with each step.

\section{B. Trajectory Generation}

Once the plane of motion has been identified at the beginning of each step cycle, appropriate task space trajectories must be generated for the COM $\left(x_{C O M}\right)$ and the swing foot $\left(x_{S W I N G}\right)$.

In the 2D case, the main goal of the initial states PUSH, LIFT and SWING was to achieve enough forward motion to destabilize the biped. In the $3 \mathrm{D}$ case, the robot must also remain stable in the non-motion plane while achieving the desired movement in the plane of motion. If the ZMP leaves the region of support formed by the stance foot as the swing foot is lifted, the biped begins to fall in the non-motion plane and the solution to the 2D FPE equation is insufficient to maintain stable gait. To ensure both forward progress and non-motion plane stability, the trajectories for the $x_{C O M}$ are generated as follows:

PUSH $x_{C O M}$ is moved above the leading stance foot to maintain stability in the non-motion and motion planes.

LIFT $x_{C O M}$ is held at its current location (above stance foot) while the swing foot is lifted from the ground to achieve sufficient clearance

SWING $x_{C O M}$ is held in place until the swing foot is aligned with the stance foot in the non-motion plane. At this point the $x_{C O M}$ is deliberately pushed outside the region of support in the plane of motion direction.

A similar approach is used to generate trajectories for $x_{S W I N G}$ to achieve the desired behaviour of generating enough momentum to destabilize the biped in the plane of motion while maintaining stability in the non-motion plane. Trajectories for $x_{S W I N G}$ are always computed to align with the plane of motion formed by the stance foot at the start of the step cycle. This ensures that the solution to the 2D FPE equation remains valid as the DROP state is entered.

PUSH $x_{S W I N G}$ is held in place as the $x_{C O M}$ trajectory is tracked.

LIFT $x_{S W I N G}$ follows a ramped trajectory to simultaneously raise the foot off the ground and move it forward in the plane of motion

SWING $x_{S W I N G}$ follows a straight line trajectory at a specific ground clearance until it reaches the FPE angle $\phi$

The ramp trajectory used to raise the swing foot during LIFT should be parameterized in terms of the velocity of the FPE point so that this state transitions faster in the event of larger disturbances (since the biped would have a shorter amount of time to swing the foot over and catch itself).

Depending on the supervisory control mode (i.e. WALK or STAND), the swing leg trajectory can be adjusted to implicitly achieve a desired goal. During WALK mode, the swing foot trajectory tracks a point on the ground slightly behind the FPE point. This under stepping behaviour results in the biped having enough forward moving momentum when the swing foot comes in contact with the ground such that the biped is unstable. As a result, the FPE point is continuously moving forward causing the state machine to transition into the opposing foot's LIFT state upon contact. In the STAND mode, the swing foot trajectory is adjusted to overstep the FPE point so that the biped comes to a stop following this step.

\section{Control Strategy}

A hybrid control strategy is used to simultaneously maintain stability in the non-motion plane, achieve sufficient forward momentum along a selected plane of motion and ultimately track the FPE location to regain stability by taking a step. Similar to the approach presented in [11], our approach uses a state machine to transition through the step sequence with each state having a local controller.

During the initial states of the step cycle, whole body motion control is used to track the $x_{C O M}$ and $x_{S W I N G}$ trajectories described in Section III-B. To generate the corresponding joint level trajectories, the Jacobian matrix is used to map between the task space and the joint space velocities:

$$
J=\left[\begin{array}{ll}
\partial q_{a c t} & \partial x_{\text {base }}
\end{array}\right]_{m \times(n+6)}
$$

We utilize a prioritized task space control scheme to generate joint level trajectories which simultaneously achieve state goals while satisfying the highest priority constraint (i.e. holding the $x_{C O M}$ position). The state-dependent joint level trajectories can be computed by projecting the lower priority task space goals onto the null space of higher priorities:

$$
\dot{q}_{r e f}=S\left(J_{H}^{\#} \dot{x}_{H}+N_{H} J_{L}^{\#} \dot{x}_{L}\right)
$$

Where, $S=\left[\begin{array}{ll}I_{n \times n} & 0_{n \times 6}\end{array}\right]$ is the actuator selection matrix for (4), $J^{\#}$ is the pseudoinverse of the Jacobian $J, \dot{q}_{r e f}$ is the reference joint velocity, and $\dot{x}_{H}$ and $\dot{x}_{L}$ are the high and low priority task space velocities, respectively. $J_{H}$ are $J_{L}$ are the corresponding high and low priority Jacobians, and $N_{H}=I-J_{H}^{\#} J_{H}$ is the null space projection matrix. The reference joint velocities are integrated to obtain the reference command signal to be tracked by high gain local PD controllers. The specific prioritization of each state is discussed in Section III-D.

When the biped enters the terminal state, our hybrid control strategy switches to directly computing the joint level commands using inverse kinematics. The PD controller gains of the stance foot ankle are set to $K_{P}=K_{D}=0$ to allow the biped to pivot and fall forward. Simultaneously, the inverse kinematics for the swing leg is solved directly to track the FPE point along the selected plane of motion.

\section{State Dependent Controllers}

This section presents the specific controller formulation used during each state of the gait cycle. 
1) STAND: The goal during this state is to maintain the $\mathrm{COM}$ position at the geometric centroid of both feet. In order to remain stable under small disturbances, the Jacobian under double support phase is used to compensate for the error $\Delta x_{C O M}$ in the $\mathrm{X}$ and $\mathrm{Y}$ directions.

$$
\begin{aligned}
& J_{H}=\left[\begin{array}{c}
J_{\text {Stand }} \\
J_{\text {Swing }} \\
J_{C O M}
\end{array}\right] \dot{x}_{H}=\left[\begin{array}{c}
0 \\
0 \\
\dot{x}_{C O M}
\end{array}\right] \\
& J_{L}=[0] \quad \dot{x}_{L}=[0]
\end{aligned}
$$

2) PUSH: The goal during this state is to track the trajectory generated for $x_{C O M}$ to move to the stance foot support region while remaining in the double support phase. An augmented Jacobian matrix is used to track the trajectory while simultaneously maintaining the foothold constraints.

$$
\begin{aligned}
& J_{H}=\left[\begin{array}{c}
J_{\text {Stand }} \\
J_{\text {Swing }} \\
J_{C O M}
\end{array}\right] \dot{x}_{H}=\left[\begin{array}{c}
0 \\
0 \\
\dot{x}_{C O M}
\end{array}\right] \\
& J_{L}=[0] \quad \dot{x}_{L}=[0]
\end{aligned}
$$

The joint level reference velocities are calculated from (6) and integrated to obtain the position command.

3) LIFT: In the lift stage, the highest priority task is maintaining the foothold of the stance foot, holding the $x_{C O M}$ directly above it and simultaneously raising the swing foot from the ground. The key challenge in this state is that lifting the swing foot can potentially cause the centre of pressure to leave the support region formed by the contact points of the stance foot. The prioritized task space control scheme is used to generate joint level commands to track the $x_{S W I N G}$ trajectory while satisfying the higher priority goal of maintaining the foothold and balance.

$$
\left.\begin{array}{rl}
J_{H} & =\left[\begin{array}{c}
J_{\text {Stance }} \\
J_{C O M}
\end{array}\right] \dot{x}_{H}=\left[\begin{array}{c}
0 \\
\dot{x}_{C O M}
\end{array}\right] \\
J_{L} & =\left[\begin{array}{l}
J_{\text {Swing }}
\end{array}\right] \dot{x}_{L}=\left[\dot{x}_{\text {Swing }}\right.
\end{array}\right]
$$

The joint level reference velocities are calculated from (6) and integrated to obtain the position command.

4) SWING: At this point, the goal of our control approach is to generate a forward moving momentum along the selected plane of motion. This deliberately destabilizes the biped by pushing $x_{C O M}$ outside the region of support in the chosen direction of motion. The task space prioritization in this state remains consistent with the previous state until the biped is unstable, at which point the control strategy enters the terminal DROP state.

$$
\begin{aligned}
J_{H} & =\left[\begin{array}{l}
J_{\text {Stance }} \\
J_{\text {Swing }}
\end{array}\right] \dot{x}_{H}=\left[\begin{array}{c}
0 \\
\dot{x}_{\text {Swing }}
\end{array}\right] \\
J_{L} & =\left[\begin{array}{l}
\left.J_{\text {COM }}\right] \quad \dot{x}_{L}=\left[\dot{x}_{C O M}\right.
\end{array}\right]
\end{aligned}
$$

The joint level reference velocities are calculated from (6) and integrated to obtain the position command.

5) DROP: In this terminal state, we use the Jacobian to track the fixed stance foot position and the generated swing foot trajectory to track the FPE point on the ground. Since the ZMP is outside of the region of foot support during this state, we treat the torso as a fixed base link and compute the Jacobian matrix of each foot.

$$
\begin{aligned}
J_{H} & =\left[\begin{array}{l}
J_{\text {Stance }} \\
J_{\text {Swing }}
\end{array}\right] \dot{x}_{H}=\left[\begin{array}{l}
\dot{x}_{\text {Stand }} \\
\dot{x}_{\text {Swing }}
\end{array}\right] \\
J_{L} & =[0] \quad \dot{x}_{L}=[0]
\end{aligned}
$$

By the assumption of an arbitrary 3D biped with finite sized feet, it is possible for the biped to land on the edge of the foot instead of landing perfectly above the FPE point on teh ground. To handle this behaviour, we switch to a stabilization substate where the joint level control is computed directly. At this point, we generate trajectories for the ankles to align the surface of the foot with the ground and switch to high gain PD control for tracking. This ensures that both feet are in full contact with the ground prior to executing the opposite legs gait sequence.

\section{Simulations AND Results}

\section{A. 2D Simulations}

The compass biped model with the single state variable is simulated to illustrate the effects of overstepping and under stepping. The leg separation angle $\beta$ is held constant and no energy is lost upon impact.

When the biped under steps, the kinetic energy exceeds the potential energy and the biped remains in forward motion (as shown in Figure 4). Note that a biped using the state machine controller described above would now calculate and track a new FPE angle to restore stability. When the compass biped over steps, the system energy is not high enough to continue forward motion. Instead, the biped rocks back on its previous stance leg (as shown in Figure 5).

\section{B. $3 D$ Simulations}

A fully actuated 14 DOF lower body humanoid robot $(700 \mathrm{~mm}$ tall, $30 \mathrm{~kg}$ ) was designed as an experimental platform for bipedal locomotion research at the University of Waterloo. The proposed control strategy to extend the FPE theory to 3D was implemented in dynamic simulations on a model of the 14 DOF robot. To demonstrate the dynamic stability of a 3D biped 


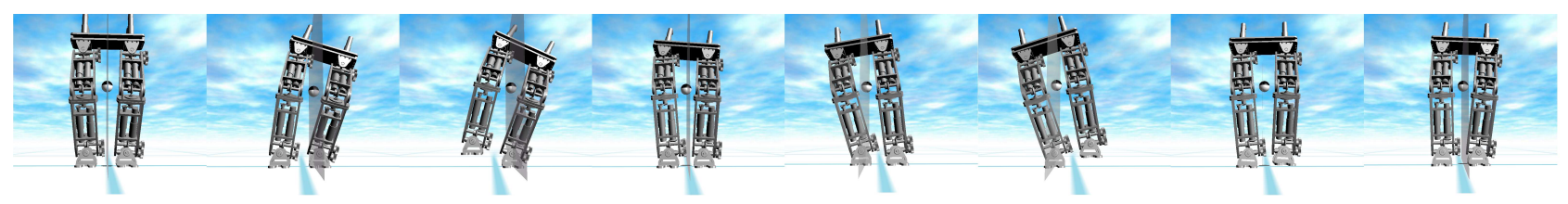

Fig. 6. Frames from the sequence of motions while side stepping. The gray plane in the frame captures above represents the $2 \mathrm{D}$ FPE plane which moves along the Y-direction (biped's frontal plane). The intersection of the gray 2D FPE plane and the ground plane indicate the FPE point tracked during DROP.
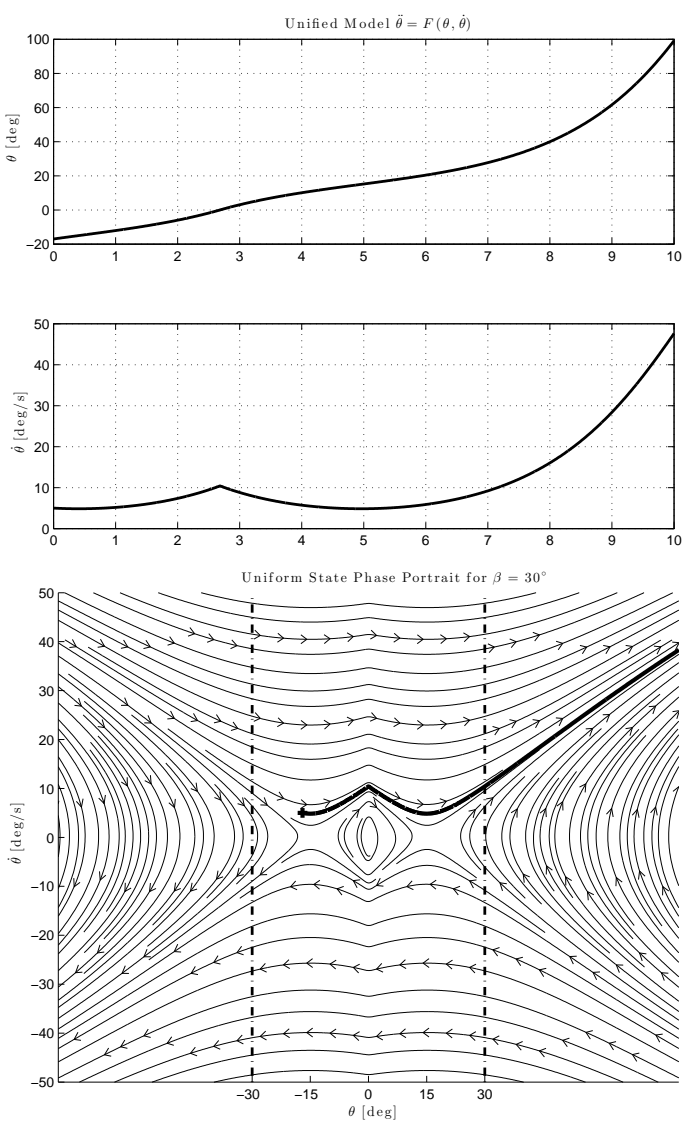

Fig. 4. Time evolution and state trajectories for under stepping (i.e. foot lands behind FPE point)

under this approach, the frontal plane was selected as the plane of motion. Forward motion along this plane results in a sideto-side stepping sequence for the biped (as shown in Figure $6)$. Each state of our control strategy was implemented in the Matlab/Simulink environment with the dynamics simulated in SimMechanics. Accurate kinematic and dynamic properties of the physical robot were taken directly from the CAD model [19].

The resulting $x_{C O M}$ trajectories from simulating the sideto-side stepping motion (shown in Figure 7) demonstrate the stability of the biped through a complete gait sequence. Our prioritized motion control framework handles the dynamic switching of constraints (from double support to single support) while generating the appropriate joint level commands for swinging the COM over. In the terminal DROP state,
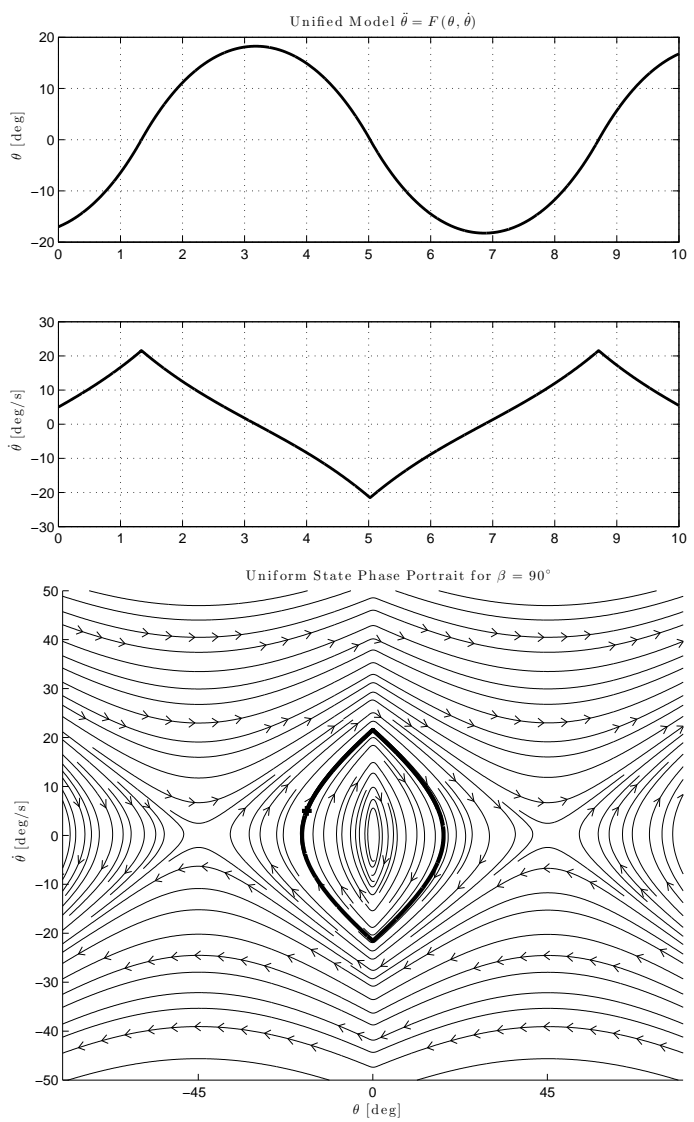

Fig. 5. Time evolution and state trajectories for over stepping (i.e. foot lands in front of FPE point)

the swing foot trajectory tracks the FPE point on the ground (shown in Figure 8) with an added offset to ensure that the biped oversteps to guarantee stability (as per the 2D FPE theory). This causes the biped to rock back and forth (similar to the $2 \mathrm{D}$ case) until stability is reached.

\section{Conclusions}

In this paper we present a method for gait generation by extending the 2D FPE algorithm for the 3D case. After selecting a plane of motion in which to generate forward momentum, we solve the 2D FPE equation to obtain the appropriate swing foot placement to maintain stability. The approach is used to form complete gait cycles using a state machine and differential kinematics. To generate the appropriate joint level trajectories to realize each state goal, we utilize 
a prioritized task space control scheme which automatically handles switching of constraints.

The simple 2D compass biped model was simulated to illustrate the effects of understepping and overstepping the FPE point. The dynamic stability of the 3D case was illustrated in simulation by completing a side-to-side stepping gait sequence. It was shown that the 3D biped remains stable by using the proposed gait generation and control strategy and overstepping the FPE point.

In future work we plan to simulate and test on a physical humanoid a variety of 3D movements including forward walking motion and responses to disturbances such as pushing by generating the plane of motion on-line.

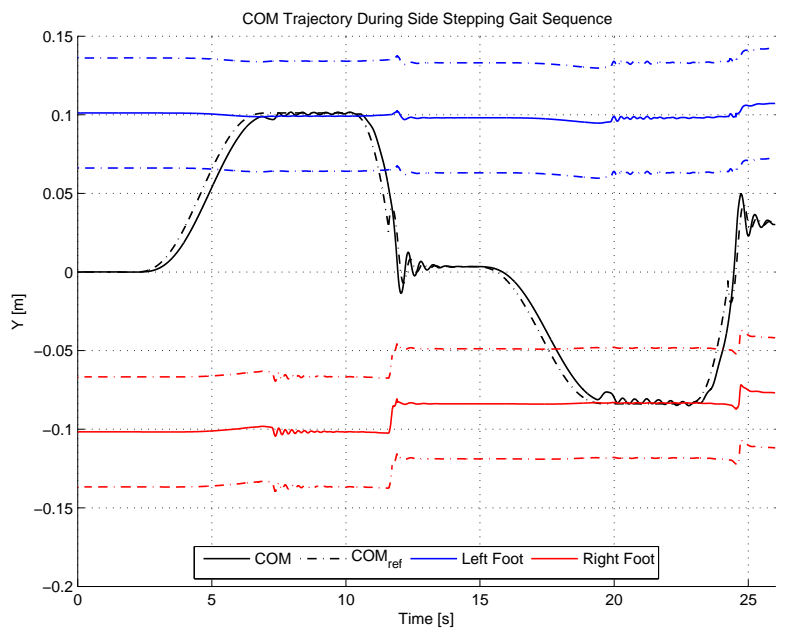

Fig. 7. COM trajectory being tracked during the complete gait sequence of side stepping. The colour coded dotted lines indicate the boundaries of each foot on the ground. Note that during the SWING state, the COM is pushed outside the region of support (around 11s). This in turn initiates the DROP state where the FPE point is tracked to regain stability.

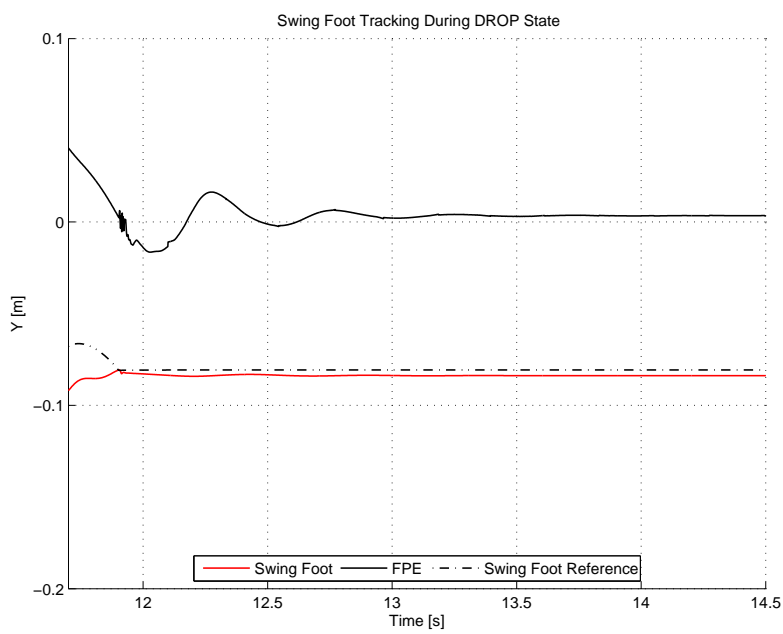

Fig. 8. During the DROP state shown here, the swing foot trajectory initially tracks the a point on the ground given by $F P E+F P E_{\text {of } f \text { set }}$ to ensure overstepping. Once ground contact is made (around 11.6s), the stabilization substate is entered and the swing foot trajectory is controlled directly to align the foot with the ground.

\section{ACKNOWLEDGMENT}

This research was supported by the Natural Sciences and Engineering Research Council of Canada.

\section{REFERENCES}

[1] S. Kajita, F. Kanehiro, K. Kaneko, K. Yokoi, and H. Hirukawa, "The 3D linear inverted pendulum mode: a simple modeling for a biped walking pattern generation," IEEE/RSJ International Conference Intelligent Robots and Systems, pp. 239-246, 2001.

[2] T. Sugihara, Y. Nakamura, and H. Inoue, "Real-time humanoid motion generation through ZMP manipulation based on inverted pendulum control," IEEE International Conference on Robotics and Automation, pp. 1404-1409, 2002.

[3] M. Vukobratovic and B. Borovac, "Zero-Moment Point - Thirty Five Years of Its Life," International Journal of Humanoid Robotics, vol. 1, no. 1, pp. 157-173, 2004.

[4] Q. Huang, K. Yokoi, S. Kajita, K. Kaneko, H. Arai, N. Koyachi, and K. Tanie, "Planning walking patterns for a biped robot," IEEE Transactions on Robotics and Automation, vol. 17, no. 3, pp. 280-289, 2001.

[5] S. Kajita, F. Kanehiro, K. Kaneko, K. Fujiwara, K. Harada, K. Yokoi, and $\mathrm{H}$. Hirukawa, "Biped walking pattern generation by using preview control of zero-moment point," in IEEE International Conference on Robotics and Automation, 2003, pp. 1620-1626.

[6] T. Takenaka, T. Matsumoto, and T. Yoshiike, "Real time motion generation and control for biped robot -1st report: Walking gait pattern generation-," in IEEE/RSJ International Conference on Intelligent Robots and Systems, 2009, pp. 1084-1091.

[7] S. Kajita, "Adaptive gait control of a biped robot based on realtime sensing of the ground profile," Autonomous Robots, vol. 4, no. 3, pp. 297-305, 1997.

[8] T. McGeer, "Passive dynamic walking," The International Journal of Robotics Research, vol. 9, no. 2, pp. 62-82, 1990.

[9] S. Collins, A. Ruina, and R. Tedrake, "Efficient bipedal robots based on passive-dynamic walkers," Science, vol. 307, no. 5712, pp. 1082-1085, 2005.

[10] M. Spong, "Passivity based control of the compass gait biped," IFAC World Congress, 1999.

[11] D. Wight, E. Kubica, and D. Wang, "Introduction of the Foot Placement Estimator: A Dynamic Measure of Balance for Bipedal Robotics," Journal of Computational and Nonlinear Dynamics, 2008.

[12] A. Goswami, B. Espiau, and A. Keramane, "Limit cycles and their stability in a passive bipedal gait," IEEE International Conference on Robotics and Automation, vol. 1, pp. 246-251, 1996.

[13] F. Asano and M. Yamakita, "Virtual passive dynamic walking and energy-based control laws," 2000, pp. 1149-1154.

[14] A. Kuo, "Stabilization of lateral motion in passive dynamic walking," The International Journal of Robotics Research, vol. 18, no. 9, pp. 917930, 1999.

[15] D. L. Wight, "A Foot Placement Strategy for Robust Bipedal Gait Control," Ph.D. dissertation, University of Waterloo, 2008.

[16] J. Pratt, J. Carff, S. Drakunov, and A. Goswami, "Capture point: A step toward humanoid push recovery," International Conference on Humanoid Robots, pp. 200-207, 2006.

[17] T. De Boer, "Foot placement in robotic bipedal locomotion," Ph.D. dissertation, Delft University, 2012.

[18] J. Pratt, T. Koolen, T. de Boer, J. Rebula, S. Cotton, J. Carff, M. Johnson, and P. Neuhaus, "Capturability-based analysis and control of legged locomotion, part 2: Application to m2v2, a lower-body humanoid," The International Journal of Robotics Research, vol. 31, no. 10, pp. 11171133, 2012.

[19] S. Choudhury, D. Wight, and D. Kulić, "Rapid prototyping toolchain for humanoid robotics applications," in IEEE International Conference on Humanoid Robots, 2012, pp. 817-822. 